\title{
Some Reduction and Exact Solutions of a Higher-Dimensional Equation
}

\author{
Guangming Wang ${ }^{1}$ and Zhong Han ${ }^{2}$ \\ ${ }^{1}$ College of Teacher Education, Tianjin Normal University, Tianjin 300387, China \\ ${ }^{2}$ College of Sciences, China University of Mining and Technology, Xuzhou 221116, China \\ Correspondence should be addressed to Guangming Wang; wanggmtj@126.com
}

Received 17 April 2014; Accepted 13 May 2014; Published 27 May 2014

Academic Editor: Huanhe Dong

Copyright (c) 2014 G. Wang and Z. Han. This is an open access article distributed under the Creative Commons Attribution License, which permits unrestricted use, distribution, and reproduction in any medium, provided the original work is properly cited.

\begin{abstract}
The conservation laws of the $(3+1)$-dimensional Zakharov-Kuznetsov equation were obtained using Noether's theorem after an interesting substitution $u=v_{x}$ to the equation. Then, with the aid of an obtained conservation law, the generalized double reduction theorem was applied to this equation. It can be verified that the reduced equation is a second order nonlinear ODE. Finally, some exact solutions of the Zakharov-Kuznetsov equation were constructed after solving the reduced equation.
\end{abstract}

\section{Introduction}

As we know that conservation laws play an important role in the study of nonlinear partial differential equations (NLPDEs) [1-3], especially in the reduction and solution of a NLPDE, an elegant and constructive way to derive conservation laws is Noether's approach $[4,5]$. The application of Noether's approach depends on a suitable Lagrangian. Yet there also exists some PDEs which do not have a Lagrangian, for example, the scalar evolution differential equations. Also there have several methods which do not relay on the knowledge of a Lagrangian. The most recent approach, due to Kara and Mahomed, is partial Noether's approach [6]. It works like the Noether's approach for PDEs with or without a Lagrangian. Different approaches to construct conservation laws were discussed in detail [7].

The fundamental relationship between symmetries and conservation laws of a PDE was given out by Kara and Mahomed in [8]. For the case of a PDE with two independent variables, using the definition of the association of symmetries with conservation laws, Sjöberg developed a theorem called double reduction method [9] which leads from a $q$ th order PDE to a $q-1$ th order ordinary differential equation (ODE). Recently, this method has been generalized in [10] and applied to a $(1+2)$-dimensional wave equation [11].
In this paper, we focus on an equation presented in [12] which shows that

$$
u_{t}+a u u_{x}+b u_{x x x}+u_{x y y}+u_{x z z}=0 .
$$

In [12], the authors investigated an isothermal multicomponent magnetized plasma and firstly derived this equation. In a very recent paper [13], the authors firstly studied the symmetry group of this equation. The one-, two-, and threeparameter optimal systems of group-invariant solutions were also given out. Then, based on the obtained optimal system, they derived the reductions and some new solutions of this equation.

This paper is arranged as follows: in Section 2, we first briefly present some notation and pertinent results which will be used in this paper. In Section 3, the conservation laws of this equation will be constructed by using Noether's approach after an interesting substituting. Then in Section 4, with the aid of an obtained conservation law and its associated symmetries, the generalized double reduction method will be applied to the Zakharov-Kuznetsov equation. And in Section 5, some exact solutions will be constructed after solving the corresponding reduced nonlinear ODE. Finally, some conclusions and discussions are given in Section 6. 


\section{Notation and Preliminaries}

We briefly present the notation and pertinent results which we utilize below. In this section, the summation convention is used whenever appropriate.

2.1. Fundamental Operators and Their Relationship. Consider that a $k$-order system of PDEs with $n$ independent variables $x=\left(x^{1}, x^{2}, \ldots, x^{n}\right)$ and $m$ dependent variables $u=$ $\left(u^{1}, u^{2}, \ldots, u^{m}\right)$ reads

$$
E_{\alpha}\left(x, u, u_{(1)}, \ldots, u_{(k)}\right)=0, \quad \alpha=1,2, \ldots, m
$$

which is assumed to be of maximal rank and locally solvable.

The collection of $r$ th-order derivatives is denoted by $u_{(r)}$, and the derivatives of $u^{\alpha}$ with respect to $x^{i}$ are $u_{i}^{\alpha}=D_{i}\left(u^{\alpha}\right)$, $u_{i j}^{\alpha}=D_{j} D_{i}\left(u^{\alpha}\right)$, where

$$
D_{i}=\frac{\partial}{\partial x^{i}}+u_{i}^{\alpha} \frac{\partial}{\partial u^{\alpha}}+u_{i j}^{\alpha} \frac{\partial}{\partial u_{j}^{\alpha}}+\cdots, \quad i=1,2, \ldots, n
$$

is the total derivative operator with respect to $x^{i}$.

The following results are well known which can be found in many literatures.

The Euler-Lagrange operator is defined as

$$
\frac{\delta}{\delta u^{\alpha}}=\frac{\partial}{\partial u^{\alpha}}+\sum_{j=1}^{\infty}(-1)^{j} D_{i_{1}} \cdots D_{i_{j}} \frac{\partial}{\partial u_{i_{1} \cdots i_{j}}^{\alpha}}, \quad \alpha=1,2, \ldots, m
$$

and the Lie-Bäcklund operator is given by

$$
X=\xi^{i} \frac{\partial}{\partial x^{i}}+\eta^{\alpha} \frac{\partial}{\partial u^{\alpha}}+\sum_{j=1}^{\infty} \zeta_{i_{1} i_{2} \cdots i_{j}}^{\alpha} \frac{\partial}{\partial u_{i_{1} \cdots i_{j}}^{\alpha}}
$$

where $\zeta_{i_{1} i_{2} \cdots i_{j}}^{\alpha}$ are determined by the following formulation:

$$
\begin{gathered}
\zeta_{i}^{\alpha}=D_{i}\left(\eta^{\alpha}\right)-u_{s}^{\alpha} D_{i}\left(\xi^{s}\right), \\
\zeta_{i_{1} i_{2} \cdots i_{j}}^{\alpha}=D_{i_{j}}\left(\zeta_{i_{1} i_{2} \cdots i_{j-1}}^{\alpha}\right)-u_{s i_{1} i_{2} \cdots i_{j-1}}^{\alpha} D_{i_{j}}\left(\xi^{s}\right), \quad j>1 .
\end{gathered}
$$

And a Lie-Bäcklund operator can also be written in characteristic form as

$$
X=\xi^{i} D_{i}+W^{\alpha} \frac{\partial}{\partial u^{\alpha}}+\sum_{j=1}^{\infty} D_{i_{1}} \cdots D_{i_{j}}\left(W^{\alpha}\right) \frac{\partial}{\partial u_{i_{1} \cdots i_{j}}^{\alpha}},
$$

where $W^{\alpha}=\eta^{\alpha}-\xi^{j} u_{j}^{\alpha}, \alpha=1,2, \ldots, m$. are the Lie characteristic functions.

A Noether operator associated with a Lie-Bäcklund operator $X$ is defined as

$$
\begin{array}{r}
N^{i}=\xi^{i}+W^{\alpha} \frac{\delta}{\delta u_{i}^{\alpha}}+\sum_{j=1}^{\infty} D_{i_{1}} \cdots D_{i_{j}}\left(W^{\alpha}\right) \frac{\delta}{\delta u_{i i_{1} \cdots i_{j}}^{\alpha}}, \\
i=1,2, \ldots, n
\end{array}
$$

in which, the Euler-Lagrange operators with respect to derivatives of $u^{\alpha}$ can be achieved by replacing $u^{\alpha}$ with the corresponding derivatives. For example, the EulerLagrange operator $\delta / \delta u_{i}^{\alpha}$ is given by

$$
\begin{array}{r}
\frac{\delta}{\delta u_{i}^{\alpha}}=\frac{\partial}{\partial u_{i}^{\alpha}}+\sum_{j=1}^{\infty}(-1)^{j} D_{i_{1}} \cdots D_{i_{j}} \frac{\partial}{\partial u_{i i_{1} \cdots i_{j}}^{\alpha}}, \\
i=1,2, \ldots, n, \quad \alpha=1,2, \ldots, m
\end{array}
$$

and the other Euler-Lagrange operators with respect to higher order derivatives can be derived in the similar way.

2.2. Conservation Law. An $n$-tuple $T=\left(T^{1}, T^{2}, \ldots, T^{n}\right), T^{i} \in$ $\vartheta, i=1,2, \ldots, n$, is a conserved vector of the system (2) if $T^{i}$ satisfies

$$
\left.D_{i} T^{i}\right|_{(2)}=0,
$$

where $\vartheta$ is the space of differential functions.

2.3. Noether's Theorem. If there exists a function $L=$ $L\left(x, u, u_{(1)}, \ldots, u_{(l)}\right), l \leq k$, such that (2) is equivalent to

$$
\frac{\delta L}{\delta u^{\alpha}}=0, \quad \alpha=1,2, \ldots, m
$$

then $L$ is called a Lagrangian of (2) and (11) is the corresponding Euler-Lagrange system.

If a Lie-Bäcklund operator $X$ defined in (5) satisfies

$$
X(L)+L D_{i}\left(\xi^{i}\right)=D_{i}\left(B^{i}\right)
$$

for some vectors $B=\left(B^{1}, B^{2}, \ldots, B^{n}\right), B^{i}\left(x^{1}, \ldots, x^{n}, u^{1}\right.$, $\left.\ldots, u^{m}\right) \in \vartheta, i=1,2, \ldots, n$, then it is a Noether symmetry generator associated with the corresponding Lagrangian $L$.

Having determined the Noether symmetry generators, conservation laws of the Euler-Lagrange system can be constructed through the following theorem in an elegant way.

Theorem 1. If a Lie-Bäcklund operator X defined in (5) is a Noether symmetry generator associated with a Lagrangian $L$ of an Euler-Lagrange system, then there corresponds a vector $T=\left(T^{1}, T^{2}, \ldots, T^{n}\right)$ with $T^{i}$ given by

$$
\begin{aligned}
T^{i}= & B^{i}-N^{i} L=B^{i}-\xi^{i} L-W^{\alpha} \frac{\delta L}{\delta u_{i}^{\alpha}} \\
& -\sum_{j=1}^{\infty} D_{i_{1}} \cdots D_{i_{j}}\left(W^{\alpha}\right) \frac{\delta L}{\delta u_{i i_{1} \cdots i_{j}}^{\alpha}}, \quad i=1,2, \ldots, n
\end{aligned}
$$

which is a conserved vector of the Euler-Lagrange system, where the characteristics $W=\left(W^{1}, W^{2}, \ldots, W^{m}\right), W^{i} \in \vartheta$ of the Noether symmetry generator are also the characteristics of the obtained conservation law.

\subsection{Generalized Double Reduction Theorem}

Definition 2 (see [8]). A Lie-Bäcklund symmetry generator $X$ defined in (5) is said to be associated with a conserved vector $T$ of the system (2) if $X$ and $T$ satisfy the following identity:

$$
X\left(T^{i}\right)+T^{i} D_{k}\left(\xi^{k}\right)-T^{k} D_{k}\left(\xi^{i}\right)=0, \quad i=1, \ldots, n .
$$


Theorem 3 (see [14]). Suppose that $X$ is any Lie-Bäcklund symmetry generator of the system (2) and $T^{i}, i=1, \ldots, n$ are the components of conserved vector of (2). Then

$$
\begin{array}{r}
T^{* i}=\left[T^{i}, X\right]=X\left(T^{i}\right)+T^{i} D_{j}\left(\xi^{j}\right)-T^{j} D_{j}\left(\xi^{i}\right) \\
i=1, \ldots, n
\end{array}
$$

also constitute the components of a conserved vector for (2); that is,

$$
\left.D_{i} T^{* i}\right|_{(2)}=0
$$

Theorem 4 (see [10]). Suppose that $D_{i} T^{i}=0$ is a conservation law of the system (2). Then, under a contact transformation, there exists function $\widetilde{T}^{i}$ such that $J D_{i} T^{i}=\widetilde{D}_{i} \widetilde{T}^{i}$, where $\widetilde{T}^{i}$ is given by the following formula:

$$
\begin{gathered}
\left(\begin{array}{c}
\widetilde{T}^{1} \\
\widetilde{T}^{2} \\
\vdots \\
\widetilde{T}^{n}
\end{array}\right)=J\left(A^{-1}\right)^{T}\left(\begin{array}{c}
T^{1} \\
T^{2} \\
\vdots \\
T^{n}
\end{array}\right), \\
J\left(\begin{array}{c}
T^{1} \\
T^{2} \\
\vdots \\
T^{n}
\end{array}\right)=A^{T}\left(\begin{array}{c}
\widetilde{T}^{1} \\
\widetilde{T}^{2} \\
\vdots \\
\tilde{T}^{n}
\end{array}\right)
\end{gathered}
$$

in which

$$
\begin{gathered}
A=\left(\begin{array}{cccc}
\widetilde{D}_{1} x_{1} & \widetilde{D}_{1} x_{2} & \cdots & \widetilde{D}_{1} x_{n} \\
\widetilde{D}_{2} x_{1} & \widetilde{D}_{2} x_{2} & \cdots & \widetilde{D}_{2} x_{n} \\
\vdots & \vdots & \vdots & \vdots \\
\widetilde{D}_{n} x_{1} & \widetilde{D}_{n} x_{2} & \cdots & \widetilde{D}_{n} x_{n}
\end{array}\right), \\
A^{-1}=\left(\begin{array}{cccc}
D_{1} \widetilde{x}_{1} & D_{1} \widetilde{x}_{2} & \cdots & D_{1} \widetilde{x}_{n} \\
D_{2} \tilde{x}_{1} & D_{2} \tilde{x}_{2} & \cdots & D_{2} \widetilde{x}_{n} \\
\vdots & \vdots & \vdots & \vdots \\
D_{n} \widetilde{x}_{1} & D_{n} \widetilde{x}_{2} & \cdots & D_{n} \widetilde{x}_{n}
\end{array}\right)
\end{gathered}
$$

and $J=\operatorname{det}(A)$.

Theorem 5 (fundamental theorem on generalized double reduction [10]). Suppose that $D_{i} T^{i}=0$ is a conservation law of the system (2). Then, under a similarity transformation of a symmetry $X$ of the system (2), there exist functions $\widetilde{T}^{i}$ such that $X$ is still a symmetry for the PDE $\widetilde{D}_{i} \widetilde{T}^{i}=0$ and

$$
\left(\begin{array}{c}
X \widetilde{T}^{1} \\
X \widetilde{T}^{2} \\
\vdots \\
X \widetilde{T}^{n}
\end{array}\right)=J\left(A^{-1}\right)^{T}\left(\begin{array}{c}
{\left[T^{1}, X\right]} \\
{\left[T^{2}, X\right]} \\
\vdots \\
{\left[T^{n}, X\right]}
\end{array}\right)
$$

in which

$$
A=\left(\begin{array}{cccc}
\widetilde{D}_{1} x_{1} & \widetilde{D}_{1} x_{2} & \cdots & \widetilde{D}_{1} x_{n} \\
\widetilde{D}_{2} x_{1} & \widetilde{D}_{2} x_{2} & \cdots & \widetilde{D}_{2} x_{n} \\
\vdots & \vdots & \vdots & \vdots \\
\widetilde{D}_{n} x_{1} & \widetilde{D}_{n} x_{2} & \cdots & \widetilde{D}_{n} x_{n}
\end{array}\right),
$$

$$
A^{-1}=\left(\begin{array}{cccc}
D_{1} \tilde{x}_{1} & D_{1} \tilde{x}_{2} & \cdots & D_{1} \tilde{x}_{n} \\
D_{2} \tilde{x}_{1} & D_{2} \tilde{x}_{2} & \cdots & D_{2} \tilde{x}_{n} \\
\vdots & \vdots & \vdots & \vdots \\
D_{n} \tilde{x}_{1} & D_{n} \tilde{x}_{2} & \cdots & D_{n} \tilde{x}_{n}
\end{array}\right) \text {, }
$$

and $J=\operatorname{det}(A)$.

Corollary 6 (necessary and sufficient condition for reduced conserved form [10]). The conserved form $D_{i} T^{i}=0$ of the system (2) can be reduced under a similarity transformation of a symmetry $X$ to a reduced conserved form $\widetilde{D}_{i} \widetilde{T}^{i}=0$ if and only if $X$ is associated with the conservation law $T$; that is, $\left.[T, X]\right|_{(2)}=0$.

Corollary 7 (generalized double reduction theorem [10]). A nonlinear system of qth order PDEs with $n$ independent and $m$ dependent variables, which admits a nontrivial conserved form that has at least one associated symmetry in every reduction from the $n$ reductions (the first step of double reduction) can be reduced to $a(q-1)$ th order nonlinear system of ODEs.

\section{Conservation Laws of \\ the Zakharov-Kuznetsov Equation}

Making a substitution $u=v_{x}$ to (1), we have

$$
v_{t x}+a v_{x} v_{x x}+b v_{x x x x}+v_{x x y y}+v_{x x z z}=0 .
$$

A Lagrangian for (21) satisfying the Euler-Lagrange equation $\delta L / \delta v=0$ is given as

$$
L=-\frac{1}{2} v_{t} v_{x}-\frac{a}{6} v_{x}^{3}+\frac{b}{2} v_{x x}^{2}+\frac{1}{2} v_{x x} v_{y y}+\frac{1}{2} v_{x x} v_{z z}
$$

where the Euler-Lagrange operator $\delta / \delta v$ is given by

$$
\begin{aligned}
\frac{\delta}{\delta v}= & \frac{\partial}{\partial v}-D_{t} \frac{\partial}{\partial v_{t}}-D_{x} \frac{\partial}{\partial v_{x}}-D_{y} \frac{\partial}{\partial v_{y}}-D_{z} \frac{\partial}{\partial v_{z}}+D_{x}^{2} \frac{\partial}{\partial v_{x x}} \\
& +D_{y}^{2} \frac{\partial}{\partial v_{y y}}+D_{z}^{2} \frac{\partial}{\partial v_{z z}}+\cdots
\end{aligned}
$$

The Lie-Bäcklund operator is (invoking (5) up to third order derivatives together with (6))

$$
\begin{aligned}
X= & \xi^{1}(t, x, y, z, v) \frac{\partial}{\partial t}+\xi^{2}(t, x, y, z, v) \frac{\partial}{\partial x} \\
& +\xi^{3}(t, x, y, z, v) \frac{\partial}{\partial y}+\xi^{4}(t, x, y, z, v) \frac{\partial}{\partial z}
\end{aligned}
$$




$$
\begin{aligned}
& +\eta(t, x, y, z, v) \frac{\partial}{\partial v}+\eta_{t}^{(1)} \frac{\partial}{\partial v_{t}}+\eta_{x}^{(1)} \frac{\partial}{\partial v_{x}}+\eta_{x x}^{(2)} \frac{\partial}{\partial v_{x x}} \\
& +\eta_{y y}^{(2)} \frac{\partial}{\partial v_{y y}}+\eta_{z z}^{(2)} \frac{\partial}{\partial v_{z z}}
\end{aligned}
$$

in which $\eta_{t}^{(1)}=D_{t}(\eta)-v_{i} D_{t}\left(\xi^{i}\right), \eta_{x}^{(1)}=D_{x}(\eta)-v_{i} D_{x}\left(\xi^{i}\right)$, $\eta_{y}^{(1)}=D_{y}(\eta)-v_{i} D_{y}\left(\xi^{i}\right)$,

$$
\begin{aligned}
& \eta_{x x}^{(2)}=D_{x}\left(\eta_{x}^{(1)}\right)-v_{i x} D_{x}\left(\xi^{i}\right), \\
& \eta_{y y}^{(2)}=D_{y}\left(\eta_{y}^{(1)}\right)-v_{i y} D_{y}\left(\xi^{i}\right), \\
& \eta_{z z}^{(2)}=D_{z}\left(\eta_{z}^{(1)}\right)-v_{i z} D_{z}\left(\xi^{i}\right) .
\end{aligned}
$$

The Lie-Bäcklund operator $X$ is a Noether symmetry generator associated with the Lagrangian $L$ (22) if there exists a vector $B=\left(B^{1}, B^{2}, B^{3}, B^{4}\right)$, such that

$$
\begin{aligned}
X(L) & +L\left[D_{t}\left(\xi^{1}\right)+D_{x}\left(\xi^{2}\right)+D_{y}\left(\xi^{3}\right)+D_{z}\left(\xi^{4}\right)\right] \\
& =D_{t}\left(B^{1}\right)+D_{x}\left(B^{2}\right)+D_{y}\left(B^{3}\right)+D_{z}\left(B^{4}\right),
\end{aligned}
$$

where $B^{1}(t, x, y, z, v), B^{2}(t, x, y, z, v), B^{3}(t, x, y, z, v)$, and $B^{4}(t, x, y, z, v)$ are the gauge terms.

The expansion of (26) yields

$$
\begin{aligned}
-\frac{1}{2} v_{x} & \eta_{t}^{(1)}-\frac{1}{2}\left(v_{t}+a v_{x}^{2}\right) \eta_{x}^{(1)}+\frac{1}{2}\left(2 b v_{x x}+v_{y y}+v_{z z}\right) \eta_{x x}^{(2)} \\
& +\frac{1}{2} v_{x x} \eta_{y y}^{(2)}+\frac{1}{2} v_{x x} \eta_{z z}^{(2)} \\
& +\left(-\frac{1}{2} v_{t} v_{x}-\frac{a}{6} v_{x}^{3}+\frac{b}{2} v_{x x}^{2}+\frac{1}{2} v_{x x} v_{y y}+\frac{1}{2} v_{x x} v_{z z}\right) \\
& \times\left(\xi_{t}^{1}+v_{t} \xi_{v}^{1}+\xi_{x}^{2}+v_{x} \xi_{v}^{2}+\xi_{y}^{3}+v_{y} \xi_{v}^{3}+\xi_{z}^{4}+v_{z} \xi_{v}^{4}\right) \\
= & B_{t}^{1}+v_{t} B_{v}^{1}+B_{x}^{2}+v_{x} B_{v}^{2}+B_{y}^{3}+v_{y} B_{v}^{3}+B_{z}^{4}+v_{z} B_{v}^{4} .
\end{aligned}
$$

Splitting (27) with respect to derivatives of $v$ results in an overdetermined system of equations for $\xi^{1}, \xi^{2}, \xi^{3}, \xi^{4}$, $\eta, B^{1}, B^{2}, B^{3}$, and $B^{4}$. The solutions of this system yield the following Noether symmetries and gauge terms:

$$
\begin{gathered}
\xi^{1}=3 c_{1} t+c_{5}, \quad \xi^{2}=c_{1} x+c_{6} t+c_{7}, \\
\xi^{3}=c_{1} y+c_{2} z+c_{3}, \quad \xi^{4}=c_{1} z-c_{2} y+c_{4}, \\
\eta=-c_{1} v+\frac{1}{a} c_{6} x+\alpha(t, z-y i)+\beta(t, z+y i), \\
B^{1}=-\frac{1}{2 a} c_{6} v+\gamma^{1}(t, x, y, z), \\
B^{2}=-\frac{1}{2}\left(\alpha_{t}+\beta_{t}\right) v+\gamma^{2}(t, x, y, z), \\
B^{3}=\gamma^{3}(t, x, y, z), \quad B^{4}=\gamma^{4}(t, x, y, z), \\
\gamma_{t}^{1}+\gamma_{x}^{2}+\gamma_{y}^{3}+\gamma_{z}^{4}=0,
\end{gathered}
$$

where $c_{i}, i=1,2, \ldots, 7$ are constants, $\alpha(t, z-y i)$ and $\beta(t, z+y i)$ are two functions of their gauge terms. We can set $\gamma^{1}(t, x, y, z)=\gamma^{2}(t, x, y, z)=\gamma^{3}(t, x, y, z)=\gamma^{4}(t, x, y, z)=0$ as they contribute to the trivial part of the conserved vector.

Invoking (13) together with the inverse transformation $v=\int u d x$ yields the following independent conservation laws of the $(3+1)$-dimensional Zakharov-Kuznetsov equation:

$$
T_{1}=\left(T_{1}^{t}, T_{1}^{x}, T_{1}^{y}, T_{1}^{z}\right), \quad\left(c_{1}=1\right)
$$

in which

$$
\begin{aligned}
T_{1}^{t}= & a t u^{3}-x u^{2}-u\left(\int u d x+y \int u_{y} d x+z \int u_{z} d x\right) \\
& -3 t u_{x}\left(b u_{x}+\int u_{y y} d x+\int u_{z z} d x\right)
\end{aligned}
$$

$T_{1}^{x}$

$$
\begin{aligned}
& =-\left(a u^{2}+2 b u_{x x}+u_{y y}+u_{z z}+\int u_{t} d x\right) \\
& \times\left(x u+\int u d x+3 t \int u_{t} d x+y \int u_{y} d x+z \int u_{z} d x\right) \\
& +\left(2 u+3 t u_{t}+x u_{x}+y u_{y}+z u_{z}\right) \\
& \times\left(2 b u_{x}+\int u_{y y} d x+\int u_{z z} d x\right) \\
& +x\left(\frac{1}{3} a u^{3}+u \int u_{t} d x-u_{x}\right. \\
& \left.\quad \times\left(b u_{x}+\int u_{y y} d x+\int u_{z z} d x\right)\right)
\end{aligned}
$$

$T_{1}^{y}$

$$
\begin{gathered}
=y\left(\frac{1}{3} a u^{3}-u_{x}\left(b u+\int u_{y y} d x+\int u_{z z} d x\right)+u \int u_{t} d x\right) \\
-u_{x y}\left(x u+\int u d x+3 t \int u_{t} d x+y \int u_{y} d x+z \int u_{z} d x\right) \\
+u_{x}\left(x u_{y}+2 \int u_{y} d x+3 t \int u_{t y} d x\right. \\
\left.+y \int u_{y y} d x+z \int u_{y z} d x\right)
\end{gathered}
$$

$T_{1}^{z}$

$$
\begin{gathered}
=z\left(\frac{1}{3} a u^{3}-u_{x}\left(b u_{x}+\int u_{y y} d x+\int u_{z z} d x\right)+u \int u_{t} d x\right) \\
-u_{x z}\left(x u+\int u d x+3 t \int u_{t} d x+y \int u_{y} d x+z \int u_{z} d x\right) \\
+u_{x}\left(x u_{z}+2 \int u_{z} d x+3 t \int u_{t z} d x\right. \\
\left.+y \int u_{y z} d x+z \int u_{z z} d x\right)
\end{gathered}
$$




$$
\begin{array}{r}
T_{2}=\left(u\left(y \int u_{z} d x-z \int u_{y} d x\right), T_{2}^{x}, T_{2}^{y}, T_{2}^{z}\right), \\
\left(c_{2}=1\right)
\end{array}
$$

in which

$$
\begin{aligned}
T_{2}^{x}= & \left(a u^{2}+2 b u_{x x}+u_{y y}+u_{z z}+\int u_{t} d x\right) \\
& \times\left(y \int u_{z} d x-z \int u_{y} d x\right) \\
& -\left(y u_{z}-z u_{y}\right)\left(2 b u_{x}+\int u_{y y} d x+\int u_{z z} d x\right) \\
T_{2}^{y}= & \frac{1}{3} a z u^{3}+z u \int u_{t} d x+u_{x y}\left(y \int u_{z} d x-z \int u_{y} d x\right) \\
& -u_{x}\left(b z u_{x}+\int u_{z} d x+y \int u_{y z} d x+z \int u_{z z} d x\right) \\
T_{2}^{z}= & -\frac{1}{3} a y u^{3}-y u \int u_{t} d x+u_{x z}\left(y \int u_{z} d x-z \int u_{y} d x\right) \\
& +u_{x}\left(b y u_{x}+\int u_{y} d x+z \int u_{y z} d x+y \int u_{y y} d x\right) \\
T_{3}= & \left(-u \int u_{y} d x, T_{3}^{x}, T_{3}^{y}, u_{x} \int u_{y z} d x-u_{x z} \int u_{y} d x\right),
\end{aligned}
$$

in which

$$
\begin{gathered}
T_{3}^{x}=u_{y}\left(2 b u_{x}+\int u_{y y} d x+\int u_{z z} d x\right) \\
-\left(a u^{2}+2 b u_{x x}+u_{y y}+u_{z z}+\int u_{t} d x\right) \int u_{y} d x \\
T_{3}^{y}=\frac{1}{3} a u^{3}+u \int u_{t} d x-u_{x}\left(b u_{x}+\int u_{z z} d x\right)-u_{x y} \int u_{y} d x \\
T_{4}=\left(-u \int u_{z} d x, T_{4}^{x}, u_{x} \int u_{y z} d x-u_{x y} \int u_{z} d x, T_{4}^{z}\right), \\
\left(c_{4}=1\right)
\end{gathered}
$$

in which

$$
\begin{aligned}
T_{4}^{x}= & u_{z}\left(2 b u_{x}+\int u_{y y} d x+\int u_{z z} d x\right) \\
& -\left(a u^{2}+2 b u_{x x}+u_{y y}+u_{z z}+\int u_{t} d x\right) \int u_{z} d x \\
T_{4}^{z}= & \frac{1}{3} a u^{3}+u \int u_{t} d x-u_{x}\left(b u_{x}+\int u_{y y} d x\right) \\
& -u_{x z} \int u_{z} d x
\end{aligned}
$$

$$
\begin{gathered}
T_{5}=\left(T_{5}^{t}, T_{5}^{x}, u_{x} \int u_{t y} d x-u_{x y} \int u_{t} d x, u_{x} \int u_{t z} d x\right. \\
\left.-u_{x z} \int u_{t} d x\right) \\
\left(c_{5}=1\right)
\end{gathered}
$$

in which

$$
\begin{gathered}
T_{5}^{t}=\frac{1}{3} a u^{3}-u_{x}\left(b u_{x}+\int u_{y y} d x+\int u_{z z} d x\right) \\
T_{5}^{x}=u_{t}\left(2 b u_{x}+\int u_{y y} d x+\int u_{z z} d x\right) \\
-\left(a u^{2}+2 b u_{x x}+u_{y y}+u_{z z}+\int u_{t} d x\right) \int u_{t} d x \\
T_{6}=\left(x u-a t u^{2}-\int u d x, T_{6}^{x}, a t u_{x} u_{y}+(x-a t u) u_{x y},\right. \\
\left.a t u_{x} u_{z}+(x-a t u) u_{x z}\right) \\
\left(c_{6}=1\right)
\end{gathered}
$$

in which

$$
\begin{aligned}
& T_{6}^{x}= x\left(a u^{2}+2 b u_{x x}+u_{y y}+u_{z z}+\int u_{t} d x\right) \\
&-\int u_{y y} d x-\int u_{z z} d x \\
&-a t\left(\frac{2}{3} a u^{3}+2 b u u_{x x}+u u_{y y}+u u_{z z}-b u_{x}^{2}\right) \\
& T_{7}=\left(-u^{2}, T_{7}^{x}, u_{x} u_{y}-u u_{x y}, u_{x} u_{z}-u u_{x z}\right) \\
&\left(c_{7}=1\right)
\end{aligned}
$$

in which

$$
\begin{gathered}
T_{7}^{x}=b u_{x}^{2}-\frac{2}{3} a u^{3}-u\left(2 b u_{x x}+u_{y y}+u_{z z}\right) \\
T_{8}=\left((\alpha+\beta) u, T_{8}^{x},(\alpha+\beta) u_{x y}-i\left(\alpha_{2}-\beta_{2}\right) u_{x}\right. \\
\left.(\alpha+\beta) u_{x z}-\left(\alpha_{2}+\beta_{2}\right) u_{x}\right)
\end{gathered}
$$

in which

$$
\begin{aligned}
T_{8}^{x}= & (\alpha+\beta)\left(a u^{2}+2 b u_{x x}+u_{y y}+u_{z z}+\int u_{t} d x\right) \\
& -\left(\alpha_{1}+\beta_{1}\right) \int u d x
\end{aligned}
$$

and $\alpha_{1}, \alpha_{2}$ are the derivatives of $\alpha(t, z-y i)$ with respect to the first and second gauge terms, respectively. The similiar case is $\beta_{1}, \beta_{2}$.

Note that (35) is a local conservation law, while the others are nonlocal conservation laws. We have verified that there exist infinite many conservation laws for the ZakharovKuznetsov equation. 


\section{Double Reduction of the Zakharov- Kuznetsov Equation via (35)}

In this section, the generalized double reduction method will be applied to the Zakharov-Kuznetsov equation by using the obtained local conservation law (35) and its associated symmetries.

In the paper [13], the authors give out the following seven infinitesimal generators of this equation:

$$
\begin{gathered}
X_{1}=\frac{\partial}{\partial x}, \quad X_{2}=\frac{\partial}{\partial y}, \quad X_{3}=\frac{\partial}{\partial z}, \quad X_{4}=\frac{\partial}{\partial t} \\
X_{5}=z \frac{\partial}{\partial y}-y \frac{\partial}{\partial z}, \quad X_{6}=a t \frac{\partial}{\partial x}+\frac{\partial}{\partial u} \\
X_{7}=x \frac{\partial}{\partial x}+y \frac{\partial}{\partial y}+z \frac{\partial}{\partial z}+3 t \frac{\partial}{\partial t}-2 u \frac{\partial}{\partial u} .
\end{gathered}
$$

We first determine which of these seven symmetries are associated with the conservation law (35) through the following formula:

$$
\begin{gathered}
X\left(\begin{array}{c}
T^{t} \\
T^{x} \\
T^{y} \\
T^{z}
\end{array}\right)-\left(\begin{array}{cccc}
D_{t} \xi^{t} & D_{x} \xi^{t} & D_{y} \xi^{t} & D_{z} \xi^{t} \\
D_{t} \xi^{x} & D_{x} \xi^{x} & D_{y} \xi^{x} & D_{z} \xi^{x} \\
D_{t} \xi^{y} & D_{x} \xi^{y} & D_{y} \xi^{y} & D_{z} \xi^{y} \\
D_{t} \xi^{z} & D_{x} \xi^{z} & D_{y} \xi^{z} & D_{z} \xi^{z}
\end{array}\right)\left(\begin{array}{c}
T^{t} \\
T^{x} \\
T^{y} \\
T^{z}
\end{array}\right) \\
+\left(D_{t} \xi^{t}+D_{x} \xi^{x}+D_{y} \xi^{y}+D_{z} \xi^{z}\right)\left(\begin{array}{c}
T^{t} \\
T^{x} \\
T^{y} \\
T^{z}
\end{array}\right)=0 .
\end{gathered}
$$

It can be shown that the associated symmetries are $X_{1}, X_{2}, X_{3}, X_{4}$, and $X_{5}$, but for simplicity we adopt the combination of $X=X_{4}+c_{1} X_{1}+c_{2} X_{2}+c_{3} X_{3}=$ $\partial / \partial t+c_{1}(\partial / \partial x)+c_{2}(\partial / \partial y)+c_{3}(\partial / \partial z)$ to reduce the conserved vector, in which $c_{1}, c_{2}$, and $c_{3}$ are three constants, where the generator $X$ has a canonical form $X=\partial / \partial q$ if

$$
\frac{d t}{1}=\frac{d x}{c_{1}}=\frac{d y}{c_{2}}=\frac{d z}{c_{3}}=\frac{d u}{0}=\frac{d r}{0}=\frac{d s}{0}=\frac{d p}{0}=\frac{d q}{1}=\frac{d w}{0}
$$

or

$$
\begin{gathered}
r=x-c_{1} t, \quad s=y-c_{2} t, \quad p=z-c_{3} t, \\
q=t, \quad w(r, s, p)=u .
\end{gathered}
$$

Using the following formula, we can achieve the reduced conserved vector

$$
\left(\begin{array}{c}
T^{r} \\
T^{s} \\
T^{p} \\
T^{q}
\end{array}\right)=J\left(A^{-1}\right)^{T}\left(\begin{array}{c}
T^{t} \\
T^{x} \\
T^{y} \\
T^{z}
\end{array}\right)
$$

in which

$$
A^{-1}=\left(\begin{array}{cccc}
D_{t} r & D_{t} s & D_{t} p & D_{t} q \\
D_{x} r & D_{x} s & D_{x} p & D_{x} q \\
D_{y} r & D_{y} s & D_{y} p & D_{y} q \\
D_{z} r & D_{z} s & D_{z} p & D_{z} q
\end{array}\right), \quad J=\operatorname{det}(A)
$$

Then the reduced conserved vector is

$$
D_{r} T^{r}+D_{s} T^{s}+D_{p} T^{p}=0
$$

where

$$
\begin{aligned}
T^{r} & =-c_{1} w^{2}+\frac{2}{3} a w^{3}-b w_{r}^{2}+w\left(2 b w_{r r}+w_{s s}+w_{p p}\right), \\
T^{s} & =w w_{r s}-w_{r} w_{s}-c_{2} w^{2}, \\
T^{p} & =w w_{r p}-w_{r} w_{p}-c_{3} w^{2}, \\
T^{q} & =w^{2} .
\end{aligned}
$$

Similarly, we can determine the associated symmetry with the reduced conserved vector (44) through the following formula:

$$
\begin{gathered}
Y\left(\begin{array}{c}
T^{r} \\
T^{s} \\
T^{p}
\end{array}\right)-\left(\begin{array}{ccc}
D_{r} \xi^{r} & D_{s} \xi^{r} & D_{p} \xi^{r} \\
D_{r} \xi^{s} & D_{s} \xi^{s} & D_{p} \xi^{s} \\
D_{r} \xi^{p} & D_{s} \xi^{p} & D_{p} \xi^{p}
\end{array}\right)\left(\begin{array}{c}
T^{r} \\
T^{s} \\
T^{p}
\end{array}\right) \\
+\left(D_{r} \xi^{r}+D_{s} \xi^{s}+D_{p} \xi^{p}\right)\left(\begin{array}{c}
T^{r} \\
T^{s} \\
T^{p}
\end{array}\right)=0 .
\end{gathered}
$$

One can verify that the associated symmetries are

$$
Y_{1}=\frac{\partial}{\partial r}, \quad Y_{2}=\frac{\partial}{\partial s}, \quad Y_{3}=\frac{\partial}{\partial p} .
$$

So we can get further reduced conserved vector by the combination of $Y=Y_{1}+d_{1} Y_{2}+d_{2} Y_{3}=\partial / \partial r+d_{1}(\partial / \partial s)+$ $d_{2}(\partial / \partial p),\left(d_{1}, d_{2}\right.$ are two constants), and the generator $Y$ has a canonical form $Y=\partial / \partial h$ if

$$
\frac{d r}{1}=\frac{d s}{d_{1}}=\frac{d p}{d_{2}}=\frac{d w}{0}=\frac{d f}{0}=\frac{d g}{0}=\frac{d h}{1}=\frac{d \phi}{0}
$$

or

$$
f=s-d_{1} r, \quad g=p-d_{2} r, \quad h=r, \quad \phi(f, g)=w .
$$

Through the following formula, we can get the reduced conserved vector:

$$
\left(\begin{array}{l}
T^{f} \\
T^{g} \\
T^{h}
\end{array}\right)=J\left(A^{-1}\right)^{T}\left(\begin{array}{c}
T^{r} \\
T^{s} \\
T^{q}
\end{array}\right)
$$

in which

$$
A^{-1}=\left(\begin{array}{ccc}
D_{r} f & D_{r} g & D_{r} h \\
D_{s} f & D_{s} g & D_{s} h \\
D_{p} f & D_{p} g & D_{p} h
\end{array}\right), \quad J=\operatorname{det}(A) .
$$

And the reduced conserved vector is

$$
D_{f} T^{f}+D_{g} T^{g}=0
$$


where

$$
\begin{aligned}
T^{f}= & \left(c_{1} d_{1}-c_{2}\right) \phi^{2}-\frac{2}{3} a d_{1} \phi^{3}+\left(d_{1} \phi_{f}+d_{2} \phi_{g}\right) \\
& \times\left(b d_{1} d_{2} \phi_{g}+\left(1+b d_{1}^{2}\right) \phi_{f}\right) \\
& -\phi\left(d_{1}\left(1+2 b d_{2}^{2}\right) \phi_{g g}+d_{2}\left(1+4 b d_{1}^{2}\right) \phi_{f g}\right. \\
& \left.+2 d_{1}\left(1+b d_{1}^{2}\right) \phi_{f f}\right), \\
T^{g}= & \left(c_{1} d_{2}-c_{3}\right) \phi^{2}-\frac{2}{3} a d_{2} \phi^{3}+\left(d_{1} \phi_{f}+d_{2} \phi_{g}\right) \\
& \times\left(b d_{1} d_{2} \phi_{f}+\left(1+b d_{2}^{2}\right) \phi_{g}\right) \\
& -\phi\left(d_{2}\left(1+2 b d_{1}^{2}\right) \phi_{f f}+d_{1}\left(1+4 b d_{2}^{2}\right) \phi_{f g}\right. \\
& \left.+2 d_{2}\left(1+b d_{2}^{2}\right) \phi_{g g}\right), \\
T^{h}=- & c_{1} \phi^{2}+\frac{2}{3} a \phi^{3}-b\left(d_{1} \phi_{f}+d_{2} \phi_{g}\right)^{2} \\
+ & \phi\left(\left(1+2 b d_{1}^{2}\right) \phi_{f f}+4 b d_{1} d_{2} \phi_{f g}+\left(1+2 b d_{2}^{2}\right) \phi_{g g}\right) .
\end{aligned}
$$

Similarly, one can determine the associated symmetry with the reduced conserved vector (52) through the same formula, here we omit. One can verify that the associated symmetries with (52) are

$$
Z_{1}=\frac{\partial}{\partial f}, \quad Z_{2}=\frac{\partial}{\partial g}
$$

So we can get further reduced conserved vector by the combination of $Z=Z_{1}+e_{1} Z_{2}=\partial / \partial f+e_{1}(\partial / \partial g),\left(e_{1}\right.$ is a constant), and the generator $Z$ has a canonical form $Z=\partial / \partial \lambda$ if

$$
\frac{d f}{1}=\frac{d g}{e_{1}}=\frac{d \phi}{0}=\frac{d \theta}{0}=\frac{d \lambda}{1}=\frac{d \psi}{0}
$$

or

$$
\theta=g-e_{1} f, \quad \lambda=f, \quad \psi(\theta)=\phi .
$$

We can achieve the reduced conserved vector as follows:

$$
D_{\theta} T^{\theta}+D_{\lambda} T^{\lambda}=0
$$

in which

$$
\begin{aligned}
T^{\theta}= & \left(c_{3}-c_{2} e_{1}-\left(d_{2}-d_{1} e_{1}\right) c_{1}\right) \psi^{2}+\frac{2}{3} a\left(d_{2}-d_{1} e_{1}\right) \psi^{3} \\
& -\left(d_{2}-d_{1} e_{1}\right)\left(1+e_{1}^{2}+b\left(d_{2}-d_{1} e_{1}\right)^{2}\right) \psi^{\prime 2} \\
& +2\left(d_{2}-d_{1} e_{1}\right)\left(1+e_{1}^{2}+b\left(d_{2}-d_{1} e_{1}\right)^{2}\right) \psi \psi^{\prime \prime},
\end{aligned}
$$

$$
\begin{aligned}
T^{\lambda}= & \left(c_{2}-c_{1} d_{1}\right) \psi^{2}+\frac{2}{3} a d_{1} \psi^{3}+\left(d_{2}-d_{1} e_{1}\right) \\
& \times\left(e_{1}-b d_{1}\left(d_{2}-d_{1} e_{1}\right)\right) \psi^{\prime 2} \\
+ & \left(\left(1+2 b d_{2}^{2}\right) d_{1}-\left(1+4 b d_{1}^{2}\right) d_{2} e_{1}\right. \\
& \left.+2\left(1+b d_{1}^{2}\right) d_{1} e_{1}^{2}\right) \psi \psi^{\prime \prime} .
\end{aligned}
$$

The last step of double reduction gives the following equation:

$$
2 A \psi \psi^{\prime \prime}-A \psi^{\prime 2}+B \psi^{3}+C \psi^{2}=D .
$$

It is a second order nonlinear ODE, in which

$$
\begin{gathered}
A=\left(d_{2}-d_{1} e_{1}\right)\left(1+e_{1}^{2}+b\left(d_{2}-d_{1} e_{1}\right)^{2}\right), \\
B=\frac{2}{3} a\left(d_{2}-d_{1} e_{1}\right), \quad C=c_{3}-c_{2} e_{1}-\left(d_{2}-d_{1} e_{1}\right) c_{1},
\end{gathered}
$$

and $D$ is a constant.

It also has $u(t, x, y, z)=\psi(\theta), \theta=\left(\left(d_{2}-d_{1} e_{1}\right) c_{1}-c_{3}+\right.$ $\left.c_{2} e_{1}\right) t-\left(d_{2}-d_{1} e_{1}\right) x-e_{1} y+z$.

\section{Exact Solutions for \\ the Zakharov-Kuznetsov Equation}

If we have solved the obtained nonlinear ODE (59), then exact solutions for the Zakharov-Kuznetsov equation can be obtained after substituting the original variables.

Note that (59) can be rewritten as

$$
\psi^{\prime \prime}=\frac{\psi^{\prime 2}}{2 \psi}-\frac{B}{2 A} \psi^{2}-\frac{C}{2 A} \psi+\frac{D}{2 A} \frac{1}{\psi} .
$$

Under the transformation $\psi^{\prime}=\chi(\psi),(55)$ becomes

$$
\chi^{\prime}(\psi)=\frac{1}{2 \psi} \chi+\left(\frac{D}{2 A} \frac{1}{\psi}-\frac{B}{2 A} \psi^{2}-\frac{C}{2 A} \psi\right) \chi^{-1},
$$

which is a Bernoulli equation. Its general solution is given by

$$
\chi^{2}(\psi)=-\frac{D}{A}+K \psi-\frac{C}{A} \psi^{2}-\frac{B}{2 A} \psi^{3}
$$

in which $K$ is an integral constant.

Recall that $\psi^{\prime}=\chi(\psi)$, so we have

$$
\psi^{\prime}(\theta)= \pm \sqrt{-\frac{D}{A}+K \psi-\frac{C}{A} \psi^{2}-\frac{B}{2 A} \psi^{3}} .
$$

Note that Fan once obtained some exact solutions for the following more general nonlinear ODE:

$$
\frac{d \phi(\xi)}{d \xi}=\varepsilon \sqrt{\sum_{i=0}^{4} c_{i} \phi^{i}},
$$

where $\varepsilon= \pm 1, c_{i}(i=0,1,2,3,4)$ are constants. 
For this knowledge, we refer readers to [15]. But for volumes, they are not presented here.

Next, exact solutions of (64) will be constructed with the aid of the obtained results by Fan, and thus they are also solutions of the Zakharov-Kuznetsov equation after substituting the original variables.

Two particular cases will be investigated as follows.

Case $1(D=K=0)$. This case can be further divided into three subclasses as follows.

(1) If $C / A<0$, we have

$$
u(t, x, y, z)=-\frac{2 C}{B} \operatorname{sech}^{2}\left(\frac{1}{2} \sqrt{-\frac{C}{A}} \theta\right) .
$$

(2) If $C / A>0$, we have

$$
u(t, x, y, z)=-\frac{2 C}{B} \sec ^{2}\left(\frac{1}{2} \sqrt{\frac{C}{A}} \theta\right) .
$$

(3) If $C=0$, we have

$$
u(t, x, y, z)=-\frac{2 A}{B \theta^{2}}
$$

Which are the bell-shape soliton solution, the periodic triangular function solution and the rational function solution, respectively.

Case $2(B / A<0)$. In this case, we achieve a solution

$$
u(t, x, y, z)=\wp\left(\frac{1}{2} \sqrt{-\frac{B}{2 A}} \theta, g_{2}, g_{3}\right),
$$

in which $g_{2}=8 A K / B, g_{3}=16 D / B$. This a Weierstrass elliptic function solution.

In all of the obtained solutions, $A=\left(d_{2}-d_{1} e_{1}\right)\left(1+e_{1}^{2}+\right.$ $\left.b\left(d_{2}-d_{1} e_{1}\right)^{2}\right), \quad B=(2 / 3) a\left(d_{2}-d_{1} e_{1}\right), \quad C=c_{3}-c_{2} e_{1}-\left(d_{2}-\right.$ $\left.d_{1} e_{1}\right) c_{1}$, and $\theta=\left(\left(d_{2}-d_{1} e_{1}\right) c_{1}-c_{3}+c_{2} e_{1}\right) t-\left(d_{2}-d_{1} e_{1}\right) x-$ $e_{1} y+z$.

Remark 8. If we substitute (64) into (61) and eliminate $\psi^{\prime}$ in (61), then we can get the following equation:

$$
\psi^{\prime \prime}(\theta)=\frac{K}{2}-\frac{C}{A} \psi-\frac{3 B}{4 A} \psi^{2} .
$$

Solving (70) can also help us find solutions to the ZakharovKuznetsov equation, which will be discussed in our future research paper.

\section{Conclusion}

In this work, in order to apply the Noether's approach to construct conservation laws for the $(3+1)$-dimensional Zakharov-Kuznetsov equation, the transformation $u=v_{x}$ is utilized. It is of interest to find that a standard Lagrangian exists for the transformed equation. Then Noether's approach is used to derive the conservation laws. Finally, conservation laws for the Zakharov-Kuznetsov equation are obtained under the inverse transformation $v=\int u d x$, which involves local and nonlocal conservation laws. Many infinite conservation laws of this equation are also obtained, which shows the strong integrability of this equation. Then, with the aid of the local conservation law, the generalized double reduction theorem is applied to the Zakharov-Kuznetsov equation. It has been shown that the reduced equation is a second order nonlinear ODE. Some exact solutions of the Zakharov-Kuznetsov equation are obtained after solving the nonlinear ODE. In addition, Xia and Xiong [16] took an interesting approach to investigate soliton solutions of nonlinear equations, which induces us to consider some exact solutions of (1).

\section{Conflict of Interests}

The authors declare that there is no conflict of interests regarding the publication of this paper.

\section{References}

[1] G. W. Bluman, A. F. Cheviakov, and S. C. Anco, Applications of Symmetry Methods to Partial Differential Equations, Springer, New York, NY, USA, 2010.

[2] X.-P. Xin and Y. Chen, "The using of conservation laws in symmetry-preserving difference scheme," Communications in Theoretical Physics, vol. 59, no. 5, pp. 573-578, 2013.

[3] X.-P. Xin, Q. Miao, and Y. Chen, "Nonlocal symmetries and exact solutions for PIB equation," Communications in Theoretical Physics, vol. 58, no. 3, pp. 331-337, 2012.

[4] E. Noether, "Invariante variations probleme," Königliche Gesellschaft der Wissenschaften zu Göttingen, Mathematisch-Physikalische Klasse, vol. 2, pp. 235-257, 1918.

[5] E. Noether, "Invariant variation problems," Transport Theory and Statistical Physics, vol. 1, no. 3, pp. 186-207, 1971.

[6] A. H. Kara and F. M. Mahomed, "Noether-type symmetries and conservation laws via partial Lagrangians," Nonlinear Dynamics, vol. 45, no. 3-4, pp. 367-383, 2006.

[7] R. Naz, F. M. Mahomed, and D. P. Mason, "Comparison of different approaches to conservation laws for some partial differential equations in fluid mechanics," Applied Mathematics and Computation, vol. 205, no. 1, pp. 212-230, 2008.

[8] A. H. Kara and F. M. Mahomed, "Relationship between symmetries and conservation laws," International Journal of Theoretical Physics, vol. 39, no. 1, pp. 23-40, 2000.

[9] A. Sjöberg, "Double reduction of PDEs from the association of symmetries with conservation laws with applications," Applied Mathematics and Computation, vol. 184, no. 2, pp. 608-616, 2007.

[10] A. H. Bokhari, A. Y. Al-Dweik, F. D. Zaman, A. H. Kara, and F. M. Mahomed, "Generalization of the double reduction theory," Nonlinear Analysis: Real World Applications, vol. 11, no. 5, pp. 3763-3769, 2010.

[11] A. H. Bokhari, A. Y. Al-Dweik, A. H. Kara, F. M. Mahomed, and F. D. Zaman, "Double reduction of a nonlinear $(2+1)$ wave equation via conservation laws," Communications in Nonlinear Science and Numerical Simulation, vol. 16, no. 3, pp. 1244-1253, 2011. 
[12] G. C. Das, J. Sarma, Y.-T. Gao, and C. Uberoi, "Dynamical behavior of the soliton formation and propagation in magnetized plasma," Physics of Plasmas, vol. 7, no. 6, pp. 2374-2380, 2000.

[13] Z. Dong, Y. Chen, and Y. Lang, "Symmetry reduction and exact solutions of the $(3+1)$-dimensional Zakharov-Kuznetsov equation," Chinese Physics B, vol. 19, no. 9, Article ID 090205, 2010.

[14] A. H. Kara and F. M. Mahomed, "A basis of conservation laws for partial differential equations," Journal of Nonlinear Mathematical Physics, vol. 9, no. 2, pp. 60-72, 2002.

[15] E. G. Fan, Integrable Systems and Computer Algebras, Science Press, Beijing, China, 2004 (Chinese).

[16] T. C. Xia and S. Q. Xiong, "Exact solutions of (2+1)-dimensional Bogoyavlenskii's breaking soliton equation with symbolic computation," Computers \& Mathematics with Applications, vol. 60, no. 3, pp. 919-923, 2010. 


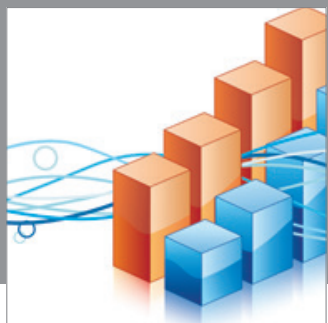

Advances in

Operations Research

mansans

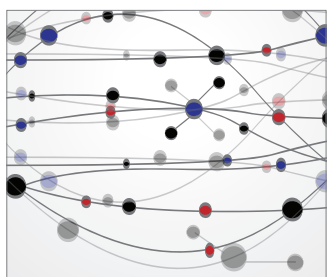

The Scientific World Journal
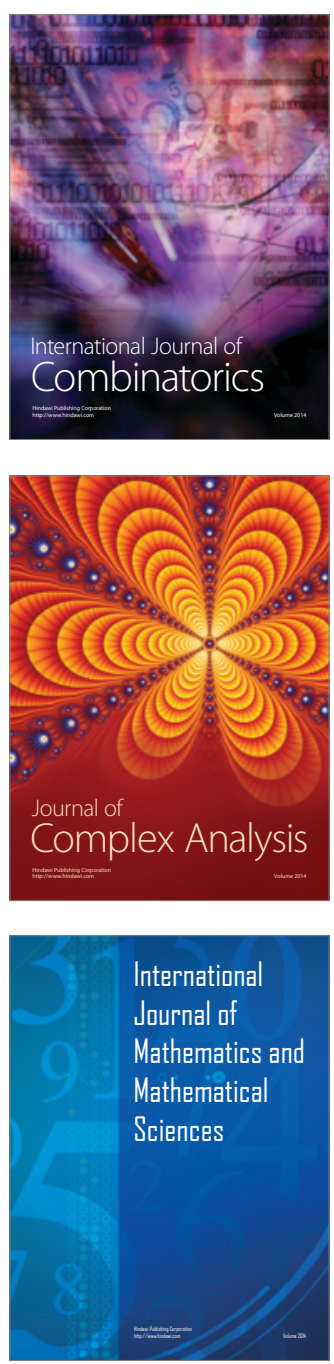
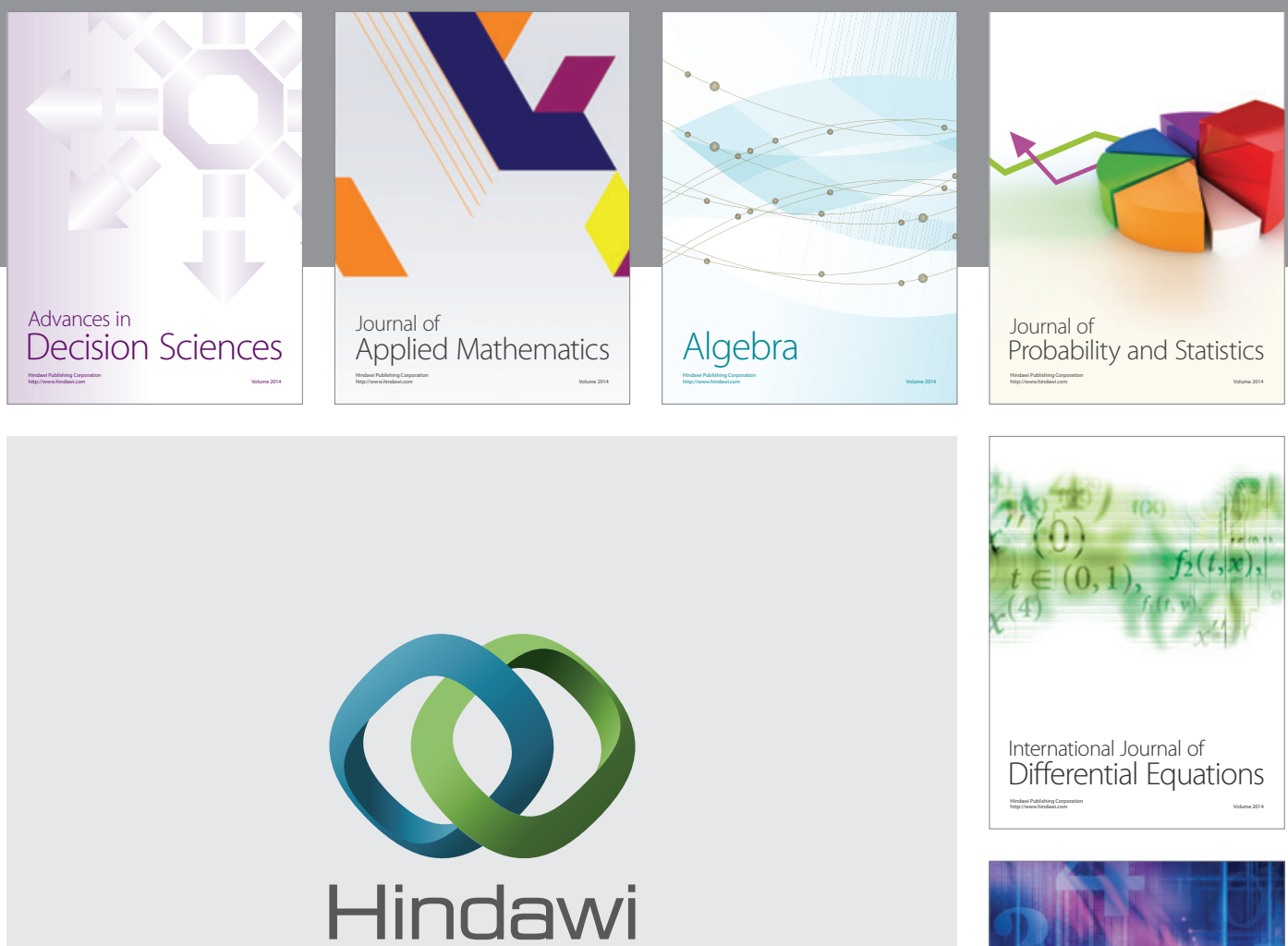

Submit your manuscripts at http://www.hindawi.com
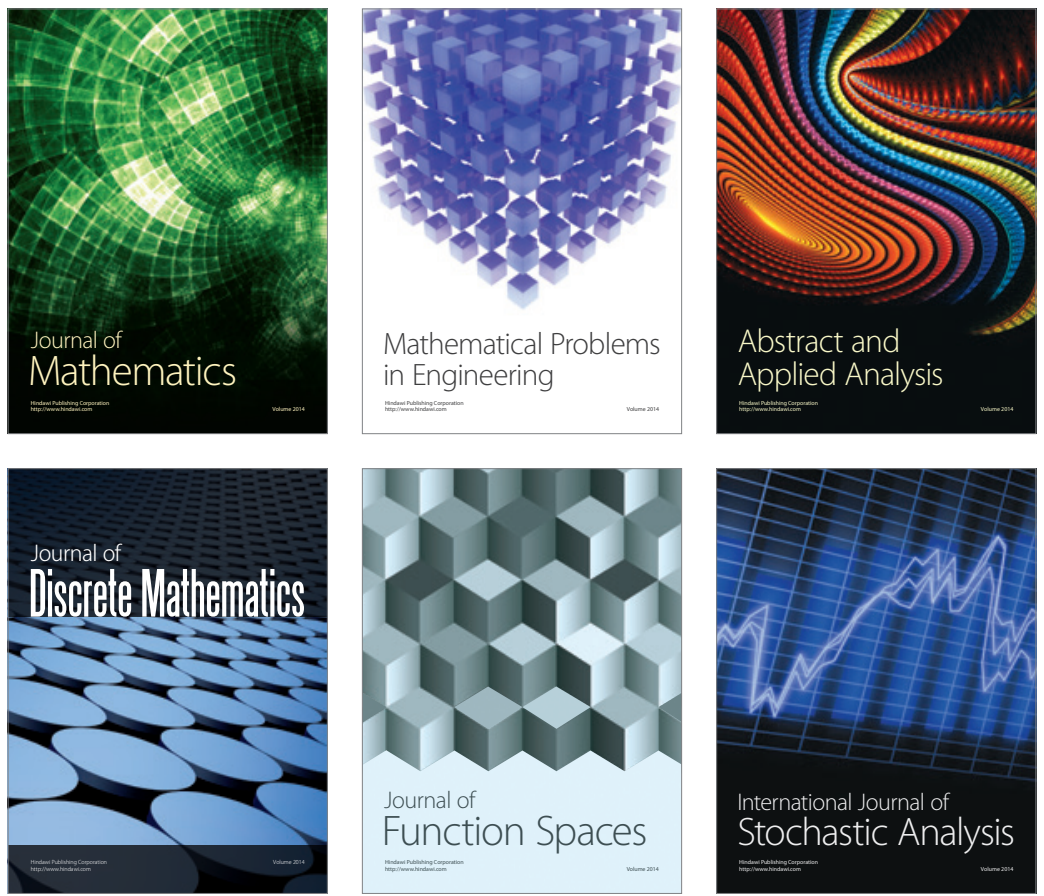

Journal of

Function Spaces

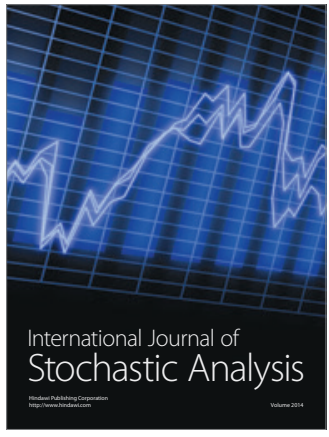

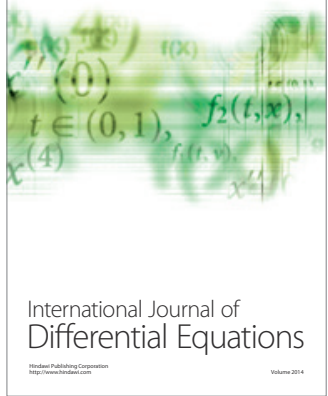
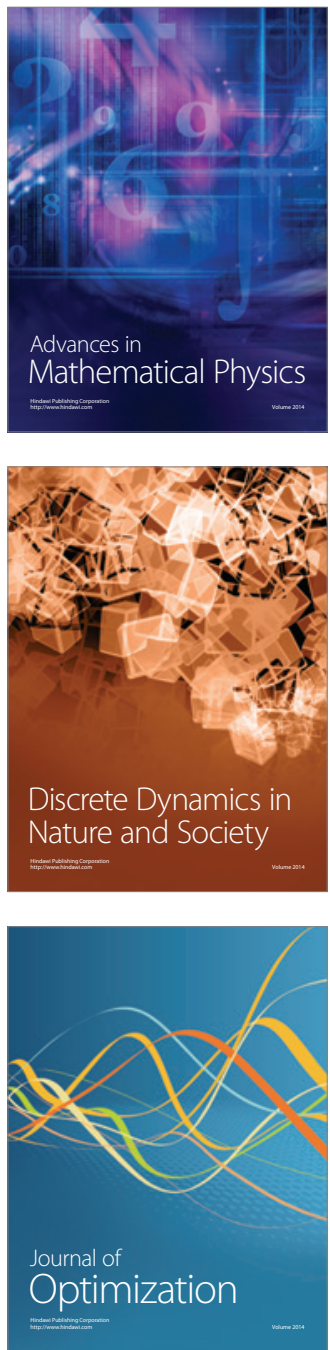